\title{
Isolating and identifying motivations: A voluntary contribution mechanism experiment with interior Nash equilibria
}

\author{
$\underline{\text { T. Kumakawa }}^{\text {a }}$, T. Saijo ${ }^{b}$ and T. Yamato ${ }^{c}$ \\ ${ }^{a}$ Institute of Social and Economic Research, Osaka University; JSPS Research Fellow PD \\ ${ }^{b}$ Research Institute for Sustainability Science, Osaka University; CASSEL, UCLA \\ ${ }^{c}$ Graduate School of Decision Science and Technology, Tokyo Institute of Technology \\ Email: kumakawa@iser.osaka-u.ac.jp
}

\begin{abstract}
A prominent finding in public good provision experiments is that contribution exceeds a noncooperative equilibrium. There are at least three possible motivations behind this overcontribution: decision errors, cooperation, and altruism. The aim of this study is to isolate and identify these motivations. The following paragraphs describe our main strategies for building our experimental model.
\end{abstract}

First, in order to discriminate cooperation from altruism, we employ a nonlinear payoff function. If we had used linear payoff functions, as done in most previous studies, such discrimination would be impossible since both cooperative and altruistic motivations appear as full investments. Using a nonlinear payoff function creates one-to-one correspondence between investments and motivations and permits us to differentiate the two motivations.

Second, in order to minimize possible decision errors, we prepare a payoff table that is comparable to the standard payoff table from previous studies. The latter is a payoff table in the nonstrategic form, or the " $N$ table," whereas the former is a payoff table in the strategic form, or the " $S$ table." The most important difference between the two payoff tables is the degree to which subjects can see the interdependence of strategies. In the case of the $N$ table, it is obscure; however, in the $S$ table, subjects can see it clearly because it is a matrix payoff table.

Third, we examine how providing information regarding the other's payoff structure to each subject affects his/her decision. Under the complete information condition, each subject knows that the other has the same payoff table as his/her own. Under the incomplete information condition, both subjects are unaware of this fact.

Since there are two payoff table conditions and two information conditions, we have four distinct treatments. We summarize the main results as follows. First, when the $S$ table was used, the average individual contributions were not statistically different from the average Nash equilibrium level. On the other hand, when the $N$ table was used, they were significantly greater than the average Nash equilibrium level. This result supports that the understanding of strategic interdependence is crucial for achieving the Nash equilibrium contribution.

Second, although the frequencies of Nash motivation were approximately $90 \%$ under the $S$ table condition, they were approximately $70 \%$ under the $N$ table condition. When subjects knew the other's payoff information, some of them showed cooperative motivation represented by the symmetric Pareto efficient contribution. Altruistic motivation that corresponded to contributing everything was rare under both conditions.

These results suggest that the cooperative and altruistic outcomes commonly observed over the past 20 years may be artifacts of the frame of the experimental environment.

Keywords: Motivations, decision errors, cooperation, altruism, voluntary contribution mechanism 


\section{INTRODUCTION}

Why people contribute to a public good even when they have economic incentives not to do so is one of the most difficult and interesting questions for economists. The last few decades have yielded a considerable number of experimental studies on the voluntary provision of a public good. When a payoff function is linear, as with most of these studies, no contribution is the dominant strategy. On the other hand, experimental results have demonstrated that subjects consistently contribute more than what is predicted by the dominant strategy and complete free riding is rare, though the average contribution gradually decays over time (see Ledyard, 1995, for a survey).

This overcontribution has led to two explanations (Andreoni, 1995). One is social preferences: subjects understand the payoff structure of the game, but still prefer to contribute rather than maximize their own payoffs. The other is decision errors: subjects do not understand the payoff structure and therefore, fail to choose the dominant strategy that would maximize their payoffs.

Beginning with Andreoni (1995), numerous attempts have been made to isolate the two explanations mentioned above. A generally accepted conclusion has been that both factors are responsible for overcontribution in linear public good experiments. Andreoni found that approximately half of overcontribution comes from social preferences and the other half from decision errors. Several other studies have also confirmed that both of these factors play leading roles in generating overcontribution (Palfrey and Prisbrey, 1997; Brandts and Schram, 2001; Goeree et al., 2002; Houser and Kurzban, 2002; Ferraro and Vossler, 2008).

However, Figure 1 indicates that the use of linear payoff functions does not allow the isolation of several motivations, since complete free riding coincides with selfish motivation, and cooperative motivation coincides with altruistic motivation. The former phenotype contribution level is zero, and the latter is all, that is, one outcome has multiple motivations.

In order to isolate these motivations, we need to use a nonlinear payoff function

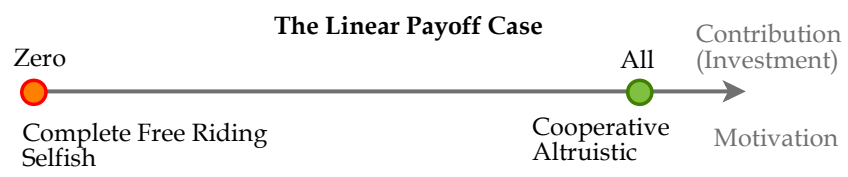
(e.g., Cobb-Douglas or quasi-linear). With nonlinear preferences, complete free riding is no longer the selfish strategy. Instead, nonzero contribution to the public good for each subject becomes a Nash equilibrium. Similarly, symmetric Pareto efficient contribution represents cooperative motivation when all subjects have the same payoff function. Furthermore, contributing one's entire endowment to the public good corresponds to altruistic motivation.

However, in the case of nonlinear public good experiments, the question regarding which motivation is important is still unsettled. A large number of studies have uniformly shown that when an interior Nash equilibrium is below the midpoint of the total endowment, as in the case of boundary equilibrium, the average contribution significantly exceeds the interior Nash equilibrium level (see Laury and Holt, 2008, for a survey). Decision errors would be canceled out in this setting because such errors appear in two different directions (above and below the equilibrium). Therefore, social preferences seem to be the source of this overprovision.

However, the types of motivations that contribute to the deviation from the standard theory are not evident in previous works. This is partially owing to experiments that do not identify the experimental environment where the theoretical prediction prevails.

Therefore, here, we attempt to minimize possible decision errors and make the experimental model follow the assumptions of the standard theory in the simplest manner possible. The following paragraphs describe the strategies adopted by us for building our experimental model.

First, we set the number of subjects at two. Most previous experiments on the provision of a public good have used at least three subjects per group. Owing to the fewer participants in the two-subject design, misunderstanding on the payoff structure can be reduced and each subject is allowed to consider only one opponent's behavior. 
Second, in order to discriminate contributions owing to decision errors, we use two types of payoff tables that are mathematically equivalent. One is a payoff table in the nonstrategic form, or the " $N$ table," and the other is a payoff table in the strategic form, or the " $S$ table." In the voluntary contribution mechanism, each subject $i$ receives the payoff from the consumption of $i$ 's private good and the payoff from the level of the public good. Then, the payoff expression is the sum of these two payoffs, which is displayed as the $N$ table. This is the type of table that was given to subjects in most of the previous experiments. The $S$ table, on the other hand, shows subject $i$ 's payoff expressed by a matrix specifying the interdependence of $i$ 's own contribution and the other's contribution to the public good.

There are two major differences between these two tables. The first difference is that the $N$ table allows subjects to clearly understand the economic framework wherein every player jointly produces a public good by their contributions; however, the $S$ table does not do so. The second difference is the degree to which subjects can see the interdependence of strategies. In the case of the $N$ table, it is obscure, whereas in the $S$ table it is obvious because it is a matrix payoff table.

Third, we examine how information regarding the other's payoff structure given to each subject, in addition to the payoff table control, affects his/her decision. Under the complete information condition, each subject knows that the other's payoff table is the same as their own table. Under the incomplete information condition, both subjects are unaware of this fact. We call this the information control.

\section{THE VOLUNTARY CONTRIBUTION MECHANISM}

There are two subjects, $a$ and $b$, and subject $i(=a, b)$ has $w_{i}$ units of the endowment of a private good. Each subject faces the decision of splitting $w_{i}$ between his/her own consumption of the private good $\left(x_{i}\right)$ and investment $\left(y_{i}\right)$ in the public good $(y)$. From the investment, each subject enjoys $y=y_{a}+y_{b}$. In other words, the level of the public good is the sum of the investments of two subjects. Therefore, the subjects' decision problem is to maximize their own payoffs, $u_{i}\left(x_{i}, y\right)$, subject to $x_{i}+y_{i}=w_{i}$. We use a quasi-linear function to transform contributions and the consumption of the private good into each subject's payoff, and all subjects have the same payoff function. We specify the payoff function as follows:

$$
u_{i}\left(x_{i}, y\right)=\alpha\left(x_{i}+\beta y-\beta y^{2} / \gamma\right)
$$

where $\left(w_{a}, w_{b}\right)=(24,24), \alpha=220, \beta=7 / 6$, and $\gamma=112$. With these parameters, the Nash equilibrium investment level is $\hat{y}=\hat{y}_{a}+\hat{y}_{b}=8$. Subjects can choose only integer investment numbers between 0 and 24; there are nine Nash equilibrium investment pairs, $\left(y_{a}, y_{b}\right)=(0,8),(1,7),(2,6), \ldots,(8,0)$. The interior Pareto efficient level of the public good, which is 32 , is also determined uniquely by the Samuelson and the feasibility conditions. Apparently, the Nash equilibrium level of the public good is less than the Pareto efficient level. The proportion of the Nash equilibrium investment to the total endowment is $8 / 48(16.7 \%)$ and the proportion of the interior Pareto efficient investment to the total endowment is 32/48 (67.7\%).

\section{EXPERIMENTAL DESIGN}

Our experiment has two parameters of control: (i) the payoff table control (the $S$ table $[S]$ vs. the $N$ table $[N]$ ), and (ii) the information control (complete information $[C]$ vs. incomplete information $[I]$ ). Thus, there are four condition pairs. Using each initial, we hereinafter refer to each of the treatments as $S C, S I, N C$, and $N I$. For example, $S C$ denotes the treatment with the $S$ table and complete information.

First, let us describe the payoff table control. We employ Table 1, which is the $N$ table, as well as the $S$ table after deleting the tag and highlighting from Table 2. Since the payoff function is quasi-linear, it can be written as $u_{i}\left(x_{i}, y\right)=u_{i}\left(w_{i}-y_{i}, y\right)=\varphi(y)+\alpha\left(w_{i}-y_{i}\right)$, where $\varphi(y)$ is the quasi-linear part of the payoff function. In Table 1, the left $1 \times 49$ table shows the $\varphi(y)$ part, and the middle saving box shows the $\alpha\left(w_{i}-y_{i}\right)$ part. Alternatively, the payoff function can be written as $u_{i}\left(w_{i}-y_{i}, y\right)=v_{i}\left(y_{i}, y_{j}\right)$ and the $S$ table expresses the payoff matrix of $v_{i}\left(y_{i}, y_{j}\right)$. Since it is easy to construct the $S$ table out of the $N$ table, let us recover the $N$ table out of the $S$ table. As an example, consider $v_{1}(4,5)=6524$. Let us raise player 1 's investment by one unit, while keeping the level of the public good at 9 . Then $v_{1}(5,4)=6304$. Therefore, the value of one unit of the private good is $220=6524-6304$, and the saving value is $4400=(24-4) \times 220$. Hence, the public good value at 9 is $2124=6524-4400$, which is the value of the public good at 9 in Table 1. It is important to note that the above procedure implicitly assumes that the target function is quasi-linear, and there is one private good and one public good. In other words, $u_{i}\left(w_{i}-y_{i}, y\right)$ cannot be recovered from $v_{i}\left(y_{i}, y_{j}\right)$ without having this economic structure. Therefore, the mathematical equivalence is valid under the 
knowledge of the structure. One of the sufficient conditions guaranteeing the interchangeability between the two payoff tables to secure their mathematical equivalence is that the payoff function is quasi-linear.

Table 1. The payoff table in the nonstrategic form (the $N$ table).

Payoff from Investment

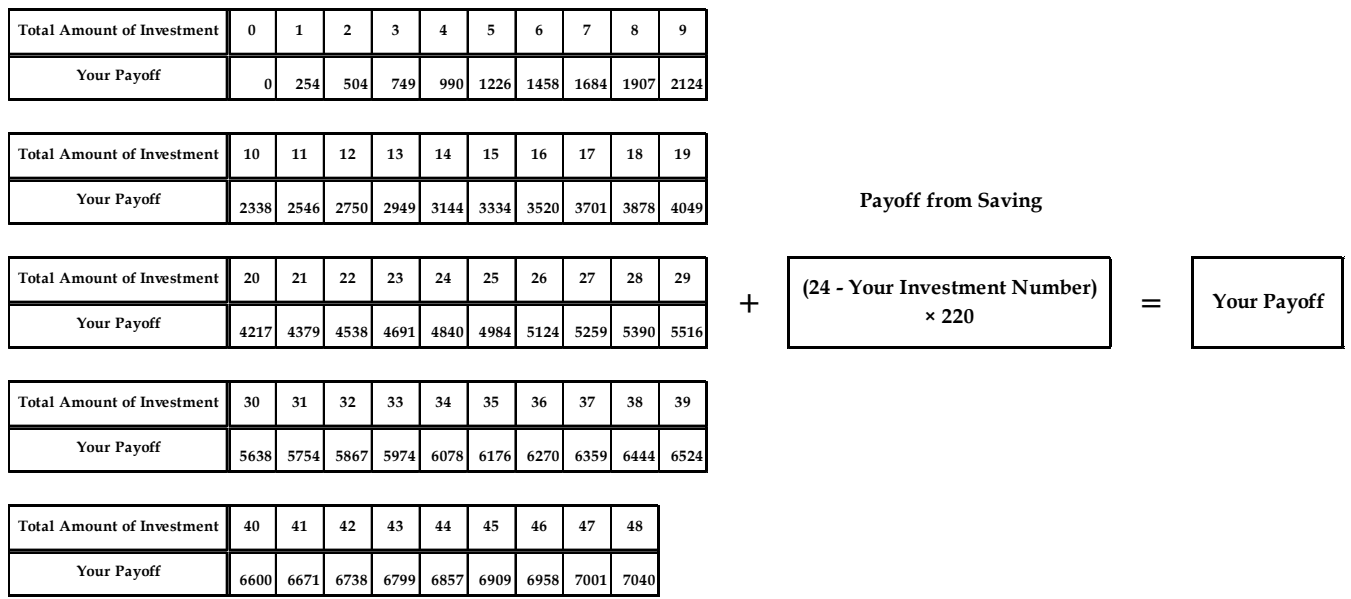

Table 2. The payoff table in the strategic form (the $S$ table).

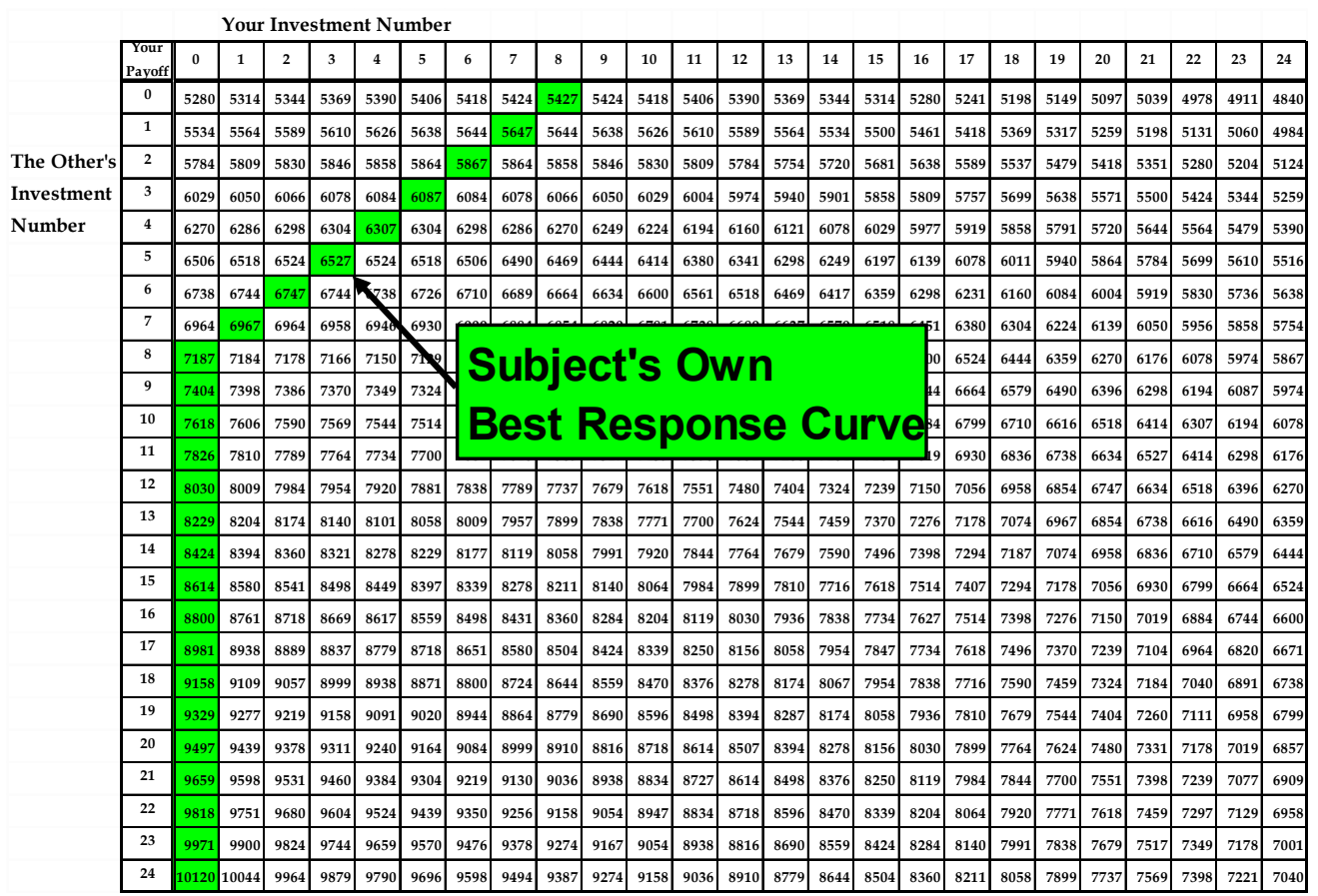

On the other hand, as Saijo and Nakamura (1995) had indicated, there are at least two qualitative differences between the two payoff tables. First, each subject can find his/her own total payoff immediately from the $S$ table, but not from the $N$ table. For example, assume that subject $a$ invests 4 and subject $b$ invests 8 . Then the total investment is 12 . By using the $N$ table, subjects can know only their respective payoffs from the public good (2750), but they have to calculate their payoffs from their private consumption for themselves. In this case, subject $a$ 's total payoff is equal to $220 \times(24-4)+2750=7150$. On the other hand, with the $S$ table, subject $a$ can immediately know his/her own total payoff by simply looking at cell $(4,8)$ in the table, where each column corresponds to each subject's own investment and each row corresponds to the other's investment.

Moreover, by simply looking at the $S$ table, subjects can compare a payoff in a certain cell with payoffs in the neighboring cells; however, subjects cannot do so with the $N$ table. The strategic interactions between subjects are visible in the $S$ table, but invisible in the $N$ table. 
Subsequently, we consider the information control. Under the complete information condition, each subject knows that the other's payoff table is exactly the same as his/her own. On the other hand, under the incomplete information condition, no subject knows the other's payoff table.

\section{RESULTS}

\subsection{Average Investment Data}

Figure 2 shows the average individual investment pattern for each treatment. First, we tested the hypothesis that the average individual investment would equal the average Nash equilibrium level (4) by pooling the data across rounds. Since the data was not independent, we considered the panel nature and used a random error specification, $v_{i t}=e_{i}+\varepsilon_{i t}$, where $e_{i}$ was a subject-specific error and $\varepsilon_{i t}$ was an IID error.

The results of the panel data analysis were as follows. In both $N C$ and $N I$, the Nash equilibrium hypothesis was rejected at the $1 \%$

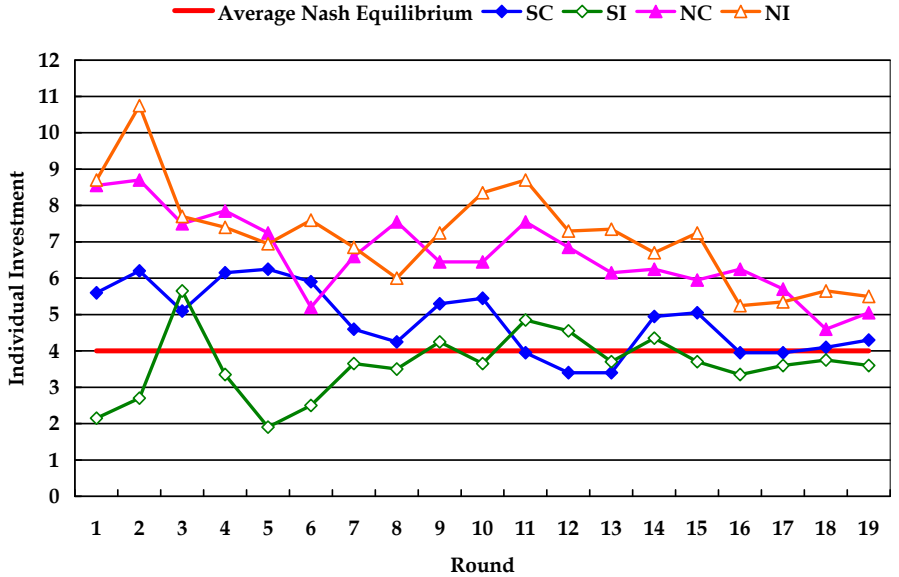

Figure 2. Average individual investment pattern for each treatment. level ( $t=3.117$ and $t=2.975$, respectively). On the other hand, in both $S C$ and $S I$, the Nash equilibrium hypothesis was not rejected at the $10 \%$ level $(t=.924$ and $t=.617$, respectively).

We also conducted round by round Wilcoxon rank-sum tests of the Nash equilibrium hypothesis. Out of a total of 19 rounds, the hypothesis was rejected in 5 rounds in $N C$ and 8 rounds in $N I$ at the $5 \%$ level. On the other hand, it was rejected in 1 round in $S C$ and 6 rounds in $S I$. Accordingly, the Nash equilibrium hypothesis was supported more frequently under the $S$ table condition.

Second, we examined the effect of each control on the average individual contribution. Using the random effects OLS regression, we compared (i) the pooled data under the $S$ table condition ( $S C$ and $S I$ ) with that under the $N$ table condition $(N C$ and $N I$ ), and (ii) the pooled data under the complete information condition ( $S C$ and $N C$ ) with that under the incomplete information condition ( $S I$ and $N I$ ). As a result, the average individual contribution under the $S$ table condition was significantly smaller than that under the $N$ table condition at the $1 \%$ level $(t=2.967)$, whereas that under the complete information condition was not statistically different from that under the incomplete information condition at the $10 \%$ level $(t=.074)$. Therefore, only the difference in the payoff tables had a significant impact on the average individual contribution, which was consistent with the results of the first regression. These results lead to the following observation:

\section{Observation 1:}

(a) Under the nonstrategic table condition, the average individual investments are significantly greater than the average Nash equilibrium level.

(b) Under the strategic table condition, the average individual investments are not statistically different from the average Nash equilibrium level.

\subsection{Identifying Motivations}

There are three focal investments in our experiment: investments " $0-8$," "16," and "24." We specify the motivation behind each investment as follows:

(i) The motivation behind investments " $0-8$ " is called "Nash motivation," since each subject chooses an investment number between 0 and 8 in every Nash equilibrium.

(ii) The motivation behind investment " 16 " is called "cooperative motivation," since by investing 16, the subject aims to achieve the cooperative outcome $(16,16)$, which is symmetrically Pareto efficient. 
(iii) The motivation behind investment "24" is called "altruistic motivation," since by investing 24 , the subject aims to maximize the other's payoff.

Note that an outcome does not always accord with each subject's motivation. For example, if subject $a$ chooses 16 with cooperative motivation, but subject $b$ chooses 4 with Nash motivation, the outcome $(16,4)$ is neither the cooperative one nor the Nash one.

Let us identify subjects' motivations using Table 3 together with Figure 1. In addition to the three investments, Table 3 also lists two intermediate investments, "9-15" and "17-23." The motivations behind these are collectively called "intermediate motivations." The frequency and percentage of each motivation were as follows.
Table 3. Frequency of individual contributions by value of contribution.

\begin{tabular}{lcccc}
\hline \multirow{2}{*}{ Contribution } & \multicolumn{4}{c}{ Treatment } \\
\cline { 2 - 5 } & $S C$ & $S I$ & $N C$ & NI \\
\hline $0-8$ & 322 & 358 & 274 & 281 \\
0 & 181 & 145 & 83 & 59 \\
$1-7$ & 46 & 140 & 134 & 117 \\
8 & 95 & 73 & 57 & 105 \\
$9-15$ & 15 & 18 & 54 & 61 \\
16 & 40 & 0 & 26 & 5 \\
$17-23$ & 0 & 1 & 21 & 25 \\
24 & 3 & 3 & 5 & 8 \\
\hline Total & 380 & 380 & 380 & 380 \\
\hline
\end{tabular}

In the SC case, "0-8" was ranked first (322, 84.7\%), "16" was second (40, 10.5\%), "9-15" was third (15, $3.9 \%)$, "24" was fourth $(3,0.8 \%)$, and " $17-23$ " had no data. In other words, the Nash and cooperative motivations accounted for over 95\% of motivations; and the Nash, cooperative, and intermediate (i.e., "9-15") motivations accounted for 99.1\%. Furthermore, altruistic motivation was rare.

In the SI case, "0-8" was ranked first $(358,94.2 \%)$, "9-15" was second $(18,4.7 \%)$, "24" was third $(3,0.8 \%)$, "17-23" was fourth $(1,0.3 \%)$, and "16" had no data. The basic motivation in this case was Nash, and the subjects did not seem to pay any attention to the cooperative outcome, since no payoff information for the other was given.

In the $N C$ case, " $0-8$ " was ranked first $(274,72.1 \%)$, "9-15" was second (54, 14.2\%), "16" was third (26, $6.8 \%)$, "17-23" was fourth $(21,5.5 \%)$, and " 24 " was fifth $(5,1.3 \%)$. In other words, the Nash, cooperative, and intermediate (i.e., "9-15") motivations accounted for over 93\% of motivations, and altruistic motivation was rare.

In the NI case, "0-8" was ranked first $(281,73.9 \%)$, “9-15" was second $(61,16.1 \%)$, "17-23" was third (25, $6.6 \%)$, "24" was fourth $(8,2.1 \%)$, and " 16 " was fifth $(5,1.3 \%)$. In other words, the Nash, cooperative, and intermediate (i.e., "9-15") motivations accounted for over $91 \%$ of motivations, and altruistic motivation was rare.

We examined the effect of each control on the frequency of each motivation. By using the Fisher's exact test, we compared (i) the pooled data under the $S$ table condition $(S C$ and $S I$ ) with that under the $N$ table condition ( $N C$ and $N I$ ); and (ii) the pooled data under the complete information condition $(S C$ and $N C$ ) with that under the incomplete information condition ( $S I$ and $N I$ ). First, a comparison of the $S$ table and $N$ table conditions revealed the frequency of Nash motivation under the $S$ table condition to be significantly greater than that under the $N$ table condition at the $1 \%$ level $(u=8.214)$. The frequencies of cooperative and altruistic motivations under the $S$ table condition were not statistically different from those under the $N$ table condition at the $10 \%$ level ( $u=1.094$ and $u=1.616$, respectively).

Second, a comparison of the complete and incomplete information conditions revealed the frequency of Nash motivation under the complete information condition to be significantly smaller than that under the incomplete information condition at the $1 \%$ level $(u=2.826)$. On the other hand, the frequency of cooperative motivation under the complete information condition was significantly greater than that under the incomplete information condition at the $1 \%$ level $(u=7.415)$. The frequency of altruistic motivation under the complete information condition was not statistically different from that under the incomplete information condition at the $10 \%$ level $(u=.693)$.

If a contribution exceeds the cooperative level (16), let us identify the motivation behind it as "quasialtruistic motivation." The ratios of quasi-altruistic motivation were $0.8 \%, 1.1 \%, 6.8 \%$, and $8.7 \%$ in $S C, S I$, $N C$, and $N I$, respectively. In other words, even by expanding the notion of altruistic motivation to the extent possible, quasi-altruistic motivation was rare under the $S$ table condition, and less than $9 \%$ under the $N$ table condition. On the other hand, although the number of observations was small, the frequency of quasi- 
altruistic motivation under the $S$ table condition was significantly smaller than that under the $N$ table condition at the $1 \%$ level $(u=6.668)$. Summarizing the above results, we have observed the following:

\section{Observation 2:}

(a) The frequency of Nash motivation under the strategic table condition is significantly greater than that under the nonstrategic table condition.

(b) The frequency of Nash motivation under the complete information condition is significantly smaller than that under the incomplete information condition.

(c) The frequency of cooperative motivation under the complete information condition is significantly greater than that under the incomplete information condition.

\section{DISCUSSION}

We have confirmed the following results.

(i) Overcontribution is observed under the nonstrategic table condition, but not under the strategic table condition.

(ii) Under the strategic table condition, there is substantially less non-Nash-motivated behavior, that is, Nashmotivated behavior accounts for approximately 90 percent of all decisions. On the other hand, under the nonstrategic table condition, Nash-motivated behavior accounts for only 73 percent of all decisions.

These results suggest that the cooperative and altruistic outcomes, which seem to dominate the experimental public good literature, may be attributable to the frame of the experimental environment.

\section{ACKNOWLEDGMENTS}

We thank the participants of the following conferences for their valuable comments and discussions: the 2009 Far East and South Asia Meeting of the Econometric Society, Fourth Asia-Pacific Meeting of the Economic Science Association, Fourth International Meeting on Experimental and Behavioral Economics, and 2008 Spring Meeting of the Japanese Economic Association. Takafumi Yamakawa, Keiko Aoki, and Masatomo Nakamura helped us conduct this experiment. This research was supported by the Grant-in-Aid for Scientific Research on Priority Areas "Experimental Social Sciences: Toward Experimentally-based New Social Sciences for the 21st Century," the 21st Century COE Program "Behavioral Macrodynamics Based on Surveys and Experiments," and the Grant-in-Aid for JSPS Fellows 211071 from the Ministry of Education, Culture, Sports, Science and Technology of Japan and the Japan Society for the Promotion of Science.

\section{REFERENCES}

Andreoni, J. (1995). Cooperation in public-goods experiments: Kindness or confusion? American Economic Review, 85, 891-904.

Brandts, J. and Schram, A. (2001). Cooperation and noise in public goods experiments: Applying the contribution function approach. Journal of Public Economics, 79, 399-427.

Ferraro, P.J. and Vossler, C.A. (2008). Stylized facts and identification in public goods experiments: The confusion confound. Georgia State University and University of Tennessee Working Paper.

Goeree, J.K., Holt, C.A., and Laury, S.K. (2002). Private costs and public benefits: Unraveling the effects of altruism and noisy behavior. Journal of Public Economics, 83, 255-276.

Houser, D. and Kurzban, R. (2002). Revisiting kindness and confusion in public goods experiments. American Economic Review, 92, 1062-1069.

Laury, S.K. and Holt, C.A. (2008). Voluntary provision of public goods: Experimental results with interior Nash equilibria. In The Handbook of Experimental Economics Results 1, Plott, C.R. and Smith, V.L. eds. (Amsterdam: North-Holland).

Ledyard, J.O. (1995). Public goods: A survey of experimental research. In The Handbook of Experimental Economics, Kagel, J.H. and Roth, A.E. eds. (Princeton, NJ: Princeton University Press).

Palfrey, T.R. and Prisbrey, J.E. (1997). Anomalous behavior in public goods experiments: How much and why? American Economic Review, 87, 829-846.

Saijo, T. and Nakamura, H. (1995). The "spite" dilemma in voluntary contribution mechanism experiments. Journal of Conflict Resolution, 39, 535-560. 